\title{
Gender differences and laterality in maximal handgrip strength and controlled force exertion in young adults
}

\author{
Hiroshi Kubota $^{1^{*}}$, Shinichi Demura ${ }^{2}$ \\ ${ }^{1}$ Faculty of Education, Gifu University, Gifu, Japan; ${ }^{*}$ Corresponding Author: hkubota@gifu-u.ac.jp \\ ${ }^{2}$ Graduate School of Natural Science \& Technology, Kanazawa University, Kanazawa, Japan.
}

Received 24 July 2011; revised 15 October 2011; accepted 3 November 2011.

\begin{abstract}
This study examines gender differences and laterality in maximal handgrip strength and controlled force exertion (CFE) in young adults. The subjects were 75 healthy young males (mean age $19.6 \pm 1.6 \mathrm{yrs}$.) and 50 healthy young females (mean age $20.9 \pm 1.9$ yrs.). Maximal handgrip strength was measured twice. The subjects performed the CFE test three times after one practice trial. They matched their handgrip strengths to the demand values, which constantly changed and ranged from 5 to $25 \%$ of maximal handgrip strength. The difference between the demand value and the grip exertion value was used as an estimate of CFE. Maximal handgrip strength was significantly larger in males than in females in both the dominant and non-dominant hands, and was significantly larger in the dominant hand in both males and females. Insignificant gender differences were found in CFE of both hands. CFE was significantly superior in the dominant hand in both genders. In conclusions, gender differences are present in maximal handgrip strength of the dominant and non-dominant hands in young adults, but not in CFE of both hands. Laterality exists in maximal handgrip strength and in CFE for both genders.
\end{abstract}

Keywords: Human; Handgrip Strength;

Neuromuscular Function

\section{INTRODUCTION}

Nerve and muscle functions are closely related to human motor performance. In particular, skillful and efficient movements that demand feedback, such as manual dexterity and hand-eye coordination, are closely involved in the coordination of the voluntary movement system, i.e. controlled force exertion (CFE) [1]. CFE is the ability to properly adjust force exertion values to demand values. Nagasawa et al. developed a CFE test in which the subjects match their grip exertion values to changing demand values on a personal computer display $[2,3]$.

Males have greater muscle strength than females [4]. Montoye and Lamphiear examined muscular abilities in males and females, and reported large gender differences in the grip and arm strengths in people aged fourteen years or older [5]. Males performed better than females in dexterity of hands and fingers involving nerve function [6-9]. York and Biederman reported that tapping test performances in males and females were almost equal [10], and Kimura et al. found no difference between males and females with respect to the agility of the body, hands and fingers [11]. Nagasawa et al. examined CFE of the dominant hand in middle-aged and elderly people and reported that males scored better than females [12]. Nagasawa et al. examined CFE of males and females aged 15 to 86 by quasi-random target-pursuit system and reported that an insignificant sex difference was found, except in subjects older than 60 years [13]. From these results, it is judged that a sex difference in of muscle strengths was found, but that the tests involving nerve function were not always conclusive.

On the other hand, a functional right and left difference, called "laterality", is found in each body part with bilateral symmetry in humans [14-17]. Laterality is inherited, and thus appears from infancy [18]. It is particularly evident in movements involving the arm or fingers, such as throwing a ball, using a spoon, or writing. According to Noguchi et al., it is found in the Beans with Tweezers test and the Pegboard test evaluating finger dexterity, and the dominant hand is found to be superior [19]. Ohtsuki et al. determined that the laterality of grading ability becomes more pronounced by the influence of an acquired factor [20]. From these researches, although laterality in the force exertion of the upper limbs is found, laterality of CFE has not yet been suffi- 
ciently examined.

It is assumed that males have greater muscle strength than females [4], gender differences are observed in CFE of the dominant hand in middle-aged and elderly people [12], that CFE of dominant hand does not show sex difference in subjects younger than 60 years old [13], and laterality exists in the upper limbs [19]. However, CFE of the non-dominant hand has not been examined, and it has not been determined whether gender difference in the dominant hand is equal to that in the non-dominant hand. The properties of muscle strength may differ from those of CFE, because muscle strength is largely affected by muscle function, while CFE is affected by both muscle and nerve functions. However, the question of whether muscle strength differs from CFE with respect to gender and laterality has not yet been sufficiently examined. This study examines CFE of young adults, but these questions need to be examined all age groups.

This study examines gender differences and laterality in maximal handgrip strength and CFE in young adults.

\section{METHODS}

\subsection{Subjects}

The subjects were 125 healthy young people consisting of 75 males (age 19.6 \pm 1.6 yrs, height $170.7 \pm 4.4$ $\mathrm{cm}$, weight $64.1 \pm 8.2 \mathrm{~kg}$ ) and 50 females (age $20.9 \pm$ $1.9 \mathrm{yrs}$, height $160.1 \pm 6.5 \mathrm{~cm}$, weight $52.7 \pm 7.0 \mathrm{~kg}$ ). No participant reported previous wrist injuries or nerve damage to upper limbs, and all were in good health. Before the experiment, all participants were judged to be right-handed by a handedness inventory survey [21]. Each subject could observe the computer display without difficulty; hence, it was concluded that individual vision did not affect measurements. No participant had previously experienced a CFE test. Prior to measurement, the purposes and procedures of this study were explained in detail, and written informed consent was obtained from all participants. The protocol of this study was approved by the Kanazawa University Department of Education Ethical Review Board.

\subsection{Test and Test Procedure}

\subsubsection{Measurement of Maximal Handgrip Strength}

Maximal handgrip strength was measured with a Smedley's handgrip mechanical dynamometer with an accuracy of $\pm 0.2 \%$ in the range of 0 to $99.9 \mathrm{~kg}$ (Sakai, Tokyo, Japan). The subjects exerted grip strength using the handgrip device while sitting in a chair with their elbow straight and close to the body. The size of the grip was set so that the subject felt comfortable squeezing it.
The maximal grip strengths of the dominant and nondominant hands were measured twice within a one-minute interval, and the greater value was used in this study.

\subsubsection{Measurement of CFE}

CFE was measured with a Smedley's handgrip mechanical dynamometer and a hand biofeedback system (EG-100; Sakai, Tokyo, Japan). The information from the handgrip device was transmitted at a sampling rate of $20 \mathrm{~Hz}$ to a computer through a data output cable after $\mathrm{A} / \mathrm{D}$ conversion. The subjects performed the CFE test while attempting to minimize the differences between the demand and grip values, as they were being presented on a computer. The display showed both demand values and actual grip values simultaneously. Relative values were used as the demand values, because the grip strength of each individual is different. The demand values changed at a constant frequency of $0.2 \mathrm{~Hz}$ from 5 to $25 \%$ of the maximal grip strength, did not regard the fatigue of subjects, and were represented by a bar graph. The difference between the demand values and the grip values was used as an estimate of CFE. A smaller difference was interpreted to mean a superior CFE. The subjects performed the CFE test in three trials using the dominant and non-dominant hands with a one-minute interval after one practice trial. The mean values of the second and third trials were used for analysis. The duration of each trial was 40 seconds, and CFE was estimated using the resulting data, excluding the first 15 seconds of each trial, in accordance with the criteria established by Nagasawa et al. $[2,3,12]$. The order of measuring the dominant and non-dominant hands was random for each subject. A sufficient rest period was given to eliminate the effect of muscle fatigue.

\subsection{Statistical Analysis}

The data are reported using ordinary statistical methods, including mean $(\mathrm{M})$ and standard deviation $( \pm$ standard deviation, SD). A two-way analysis of variance (ANOVA) was used to examine significant differences among trial means (gender and dominant/non-dominant hands). The level of significance was set a priori to 0.05 .

\section{RESULTS}

Table 1 shows the means and standard deviations of maximal handgrip strength according to the dominant and non-dominant hands in males and females, and the test results of two-way ANOVA. Gender and laterality were found to have a significant effect. Males had greater grip strength than females in both hands, and the dominant hand was greater than the non-dominant hand in both genders. 
Table 2 shows the means and standard deviations of CFE according to the dominant and non-dominant hands in males and females, and the test results of two-way ANOVA. Laterality was found to have a significant effect. An insignificant gender difference was found in CFE in both hands. The dominant hand was superior to the non-dominant hand in both genders.

\section{DISCUSSION}

Christine described that females sixteen years of age and older have about two-thirds muscle strength of males in the same age groups [4]. Moreover, these gender differences are closely correlated to differences in physiological maturation found in muscle growth. In this study, the ratio of grip strength of males and females was $67.7 \%$ in the dominant hand and $66.4 \%$ in the nondominant hand. Thus, the present results agreed with those in the above study. Christine also concluded that gender differences of muscle strength reflect the observed variation between males and females in the physical activities of daily life [4]. In short, it is inferred that handgrip strength is greatly affected also by daily life pattern.

In the present study, the dominant hand displayed maximal handgrip strength greater than that of the non-dominant hand (about $6 \%$ in males, about $8 \%$ in females). Crosby et al. similarly found that maximal handgrip strength of the dominant hand is about $6 \%$ greater than that of the non-dominant hand [22]. These results also confirm that laterality exists in both genders. The dominant hand develops as the individual habitually favors one hand in daily life $[23,24]$.
Males are superior to females in the dexterity of hands and fingers involving nerve function [6-9]. Houx examined gender differences in subjects from 20 to 80 years of age in choice reaction speed, which evaluates nerve function, and reported that males were superior to females [25]. Nagasawa et al. examined CFE of the dominant hand in middle-aged and elderly people, and saw that males were superior to females [26]. There was no gender difference found in the nerve function of the maximal ability, which involves no information feedback. However, submaximal ability, which involves information feedback, revealed gender differences.

One the other hand, Kimura et al. found no differences between males and females in the agility of body, hands and fingers [11]. Haward and Griffin did not find any sexual differences in the pegboard test. Tests which evaluate nerve function differ in their conclusions concerning gender differences [27]. Nagasawa et al. found gender differences in CFE of middle-aged and elderly people, and inferred that dexterity and speed of hands and fingers closely reflects to experiences of the movements in daily life [26]. The unique experiences of individuals create the CFE gender differences. Both young males and females have a more active daily life than the elderly, and there is no variation in their active experiences. Therefore, no difference may be discovered with respect to CFE. In addition, a gender difference was found in maximal handgrip strength, due to sex difference in muscle mass, but not in CFE, which evaluates muscle and nerve function. This may depend on the fact that, in the CFE test, subjects exert a submaximal force but not a maximal one. Maximal handgrip strength is

Table1. Means of maximal grip strength by each group and test result (two-way ANOVA).

\begin{tabular}{ccccccccc}
\hline & \multicolumn{2}{c}{ Males $(\mathrm{n}=75)$} & \multicolumn{2}{c}{ Females $(\mathrm{n}=50)$} & & \multirow{2}{*}{ F-value } & partial $\eta 2$ \\
\cline { 2 - 5 } & Mean & SD & Mean & SD & & & \\
\hline Dominant hand & 48.6 & 8.5 & 32.9 & 6.5 & F1 & $164.30^{*}$ & 0.53 \\
Non-dominant hand & 45.8 & 7.6 & 30.4 & 6.5 & F2 & $87.78^{*}$ & 0.37 \\
& & & & & F3 & 0.27 & 0.00 \\
\hline
\end{tabular}

${ }^{*} \mathrm{p}<0.05$, unit: $\mathrm{kg}, \mathrm{F} 1$ : gender, F2: dominant and non-dominant hand, F3: interaction.

Table2. Means of CFE value by each group and test result (two-way ANOVA).

\begin{tabular}{ccccccccc}
\hline & \multicolumn{2}{c}{ Males $(\mathrm{n}=75)$} & \multicolumn{2}{c}{ Females $(\mathrm{n}=50)$} & & \multirow{2}{*}{ F-value } & partial $\eta 2$ \\
\cline { 2 - 5 } & Mean & SD & Mean & SD & & & \\
\hline Dominant hand & 595.0 & 114.0 & 580.2 & 151.2 & F1 & 0.00 & 0.00 \\
Non-dominant hand & 654.0 & 110.8 & 669.4 & 136.3 & F2 & $44.89^{*}$ & 0.23 \\
& & & & & F3 & 1.94 & 0.01 \\
\hline
\end{tabular}

" $\mathrm{p}<0.05$, unit: \%, F1: gender, F2: dominant and non-dominant hand, F3: interaction. 
affected by muscle mass, but the CFE test, which uses relative values, is not largely affected by muscle mass or maximal handgrip strength.

Demura et al. found that lateral dominance appears in motor tasks which demand dexterity of the hands, fingers and upper limbs, and where the dominant hand is superior [28]. Noguchi et al. examined maximal handgrip strength and CFE in young males, and concluded that the dominant hand is easily determined by CFE, rather than by maximal handgrip strength [19]. The functions which involve motor tasks develop because of the frequent use of the dominant hand, and the developmental differences between the dominant and nondominant hands become more pronounced over time $[18,29,30]$. The present results also found that the dominant hand was superior in CFE of both genders, which agree with those in previous studies [19,21]. It is inferred that laterality is found also in the young.

\section{CONCLUSIONS}

Gender differences are found in maximal handgrip strength of the dominant and non-dominant hands in the young, but not in CFE, which involves muscle and nerve function. Laterality exists in maximal handgrip strength and CFE of both genders.

\section{REFERENCES}

[1] Henatsch, H.D. and Langer, H.H. (1985) Basic neurophysiology of motor skills in sport: A review. International Journal of Sports Medicine, 6, 2-14. doi:10.1055/s-2008-1025805

[2] Nagasawa, Y. and Demura, S. (2002) Development of an apparatus to estimate coordinated exertion of force. Perceptual \& Motor Skills, 94, 899-913.

[3] Nagasawa, Y., Demura, S. and Kitabayashi, T. (2004) Concurrent validity of tests to measure the coordinated exertion of force by computerized target pursuit. Perceptual \& Motor Skills, 98, 551-560. doi:10.2466/pms.98.2.551-560

[4] Christine, L. W. (1985) Women, sport, \& performance. Human Kinetics Publishers Ltd. Leeds.

[5] Montoye, H.J. and Lamphiear, D.E. (1977) Grip and arm strength in males and females, age 10 to 69 . Research Quarterly, 48, 109-120.

[6] Anianson, A., Rundgren, A. and Sperling, L. (1980) Evaluation of functional capacity in activities of daily living in 70-year-old men and women. Scandinavian Journal of Rehabilitation Medicine, 12, 145-154.

[7] Sperling, L. (1980) Evaluation of upper extremity function in 70-year-old men and women. Scandinavian Journal of Rehabilitation Medicine, 12, 139-144.

[8] Ruff, R.M. and Parker, S.B. (1993) Gender- and agespecific changes in motor speed and eye-hand coordination in adults: normative values for the Finger Tapping and Grooved Pegboard Tests. Perceptual \& Motor Skills, 76, 1219-1230. doi:10.2466/pms.1993.76.3c.1219
[9] Speller, L., Trollinger, J.A., Maurer, P.A., Nelson, C.E. and Bauer, D.F. (1997) Comparison of the test-retest reliability of the Work Box using three administrative methods. American Journal of Occupational Therapy, 51, 516-522. doi:10.5014/ajot.51.7.516

[10] York, J.L. and Biederman, I. (1990) Effects of age and sex on reciprocal tapping performance. Perceptual \& Motor Skills, 71, 675-684.

[11] Kimura, M., Hirakawa, K., Okuno, T., Oda, Y., Morimoto, T., Kitani, T., Fujita, D. and Nagata, H. (1989) An analysis of physical fitness in the aged people with fitness battery test. Japanese Journal of Physical Fitness and Sports Medicine, 38, 175-185.

[12] Nagasawa, Y., Demura, S., Yamaji, S., Kobayashi, H. and Matsuzawa, J. (2000) Ability to coordinate exertion of force by the dominant hand: Comparisons among university students and 65- to 78-year-old men and women. Perceptual \& Motor Skills, 90, 995-1007.

[13] Nagasawa, Y., Demura, S. and Hamazaki, H. (2010) Age and sex differences of controlled force exertion measured by a computer-generated quasi-random target-pursuit system. Journal of Musculoskeletal and Neuronal Interactions, 10, 237-244.

[14] Dolcos, F., Rice, H.J. and Cabeza, R. (2002) Hemispheric asymmentry and aging: right hemisphere decline or asymmetry reduction. Neuroscience \& Biobehavioral Reviews, 26, 819- 825 . doi:10.1016/S0149-7634(02)00068-4

[15] Geshwind, N. and Behan, P. (1982) Left-handedness: Association with immune disease, migraine, and developmental learning disorder. Proceedings of the National Academy of Sciences, 79, 5097-5100. doi:10.1073/pnas.79.16.5097

[16] Gur, R.C., Turetsky, B.I., Matsui, M., Yan, M., Bilker, W., Hughett, P. and Gur, R.E. (1999) Sex differences in brain gray and white matter in health young adults: Correlations with cognitive performance. Journal of Neuroscience, 19, 4065- 4072.

[17] Roy, E.A., Bryden, P. and Cavill, S. (2003) Hand differences in pegboard performance through development. Brain and Cognition, 53, 315-317. doi:10.1016/S0278-2626(03)00133-7

[18] Chi, J.G., Dooling, E.C. and Gilles, F.H. (1977) Left-right asymmetry of the temporal speech areas of the human fetus. Archives of Neurology, 34, 346-348.

[19] Noguchi, T., Demura, S. and Aoki, H. (2009) Superiority of dominant and nondominant hands in static strength and controlled force exertion. Perceptual \& Motor Skills, 109, 339-346. doi:10.2466/pms.109.2.339-346

[20] Ohtsuki, H., Hasebe, S., Okano, M. and Furuse, T. (1997) Comparison of surgical results of responders and nonresponders to prism adaptation test in intermittent exotropia. Acta Ophthalmol Scand, 75, 528-531. doi:10.1111/j.1600-0420.1997.tb00143.x

[21] Demura, S., Sato. S. and Nagasawa, Y. (2009) Re-examination of useful items for determining hand dominance. Medica Italiana Archivio per le Scienze Mediche, 168, 169-177.

[22] Crosby, C.A., Wehbe, M.A. and Mawr, B. (1994) Hand strength: normative values. Journal of Hand Surgery, 19, 665-670. doi:10.1016/0363-5023(94)90280-1

[23] Aoki, H. and Demura, S. (2008) The effect of gender and 
lateral dominance on gripping muscle power in young adults. Sports Science for Health, 3, 1-6. doi:10.1007/s11332-008-0054-6

[24] Aoki, H. and Demura, S. (2009) Laterality of hand grip and elbow flexion power in young male and female adults. International Journal of Sports Physiology and Performance, 4, 355-366.

[25] Houx, P.J. and Jolles, J. (1993) Age-related decline of psychomotor speed: Effects of age, brain health, sex, and education. Perceptual \& Motor Skills, 76, 195-211. doi:10.2466/pms.1993.76.1.195

[26] Nagasawa, Y., Demura, S., Yamaji, S. and Shimada, S. (2001) Relationship between physical fitness and ability to coordinate exertion of force with reference to sex difference in healthy middle- and old-aged people. Japa- nese Journal of Physical Fitness and Sports Medicine, $\mathbf{5 0}$ 425-436.

[27] Haward, B.M. and Griffin, M.J. (2002) Repeatability of grip strength and dexterity tests and the effects of age and gender. International Archives of Occupational and Environmental Health, 75, 111-119.

[28] Demura, S., Yamaji, S., Goshi, F. and Nagasawa, Y. (2001) Lateral dominance of legs in maximal muscle power, muscular endurance, and grading ability. Perceptual \& Motor Skills, 93, 11-23.

[29] Annett, M. (1975) Hand preference and the laterality of cerebral speech. Cortex, 11, 305-328.

[30] Yamamoto, T. (2001) For practical and scientific conditioning measurement and assessment. Tokyo Book House HD, Tokyo. 